\title{
1 Metamorphosis imposes variable constraints on genome expansion
}

3 Article type: Major Article

4 Keywords: genome size, constraint, development, comparative methods, life history

5 Word Count: 7,729

6 Figures: 2

7 Tables: 4 


\section{Abstract}

11 Genome size varies 100,000-fold across eukaryotes. Genome size is heavily shaped

12 by transposable element accumulation, the dynamics of which are increasingly well un-

13 derstood. However, given that traits like cell size and rate of development co-vary

14 strongly with genome size, organism-level trait evolution likely shapes genome size di-

15 versity as well. Metamorphosis - a radical transformation of morphology — has been

16 hypothesized to impact genome size because it can be a vulnerable part of the life cy-

17 cle. Thus, selection may act to limit metamorphic duration, indirectly constraining the

18 rate of development as well as genome and cell sizes. Salamanders have large and

19 variable genomes - 3 to 40 times that of humans - and species exhibit a range of

20 metamorphic and non-metamorphic life histories. Using salamanders, we test the hy-

21 pothesis that different types of metamorphic repatterning during the life cycle impose

22 different constraints on genome expansion. We show that metamorphosis during which

23 animals are unable to feed imposes the most severe constraint against genome expan-

24 sion. Other types of metamorphosis that differ in energetic provisioning impose less se-

25 vere constraints. More generally, our work demonstrates the utility of phylogenetic com-

26 parative methods in testing the role of constraint in shaping phenotypic evolution. 


\section{Introduction}

31 Across the tree of life, few characters exhibit the tremendous scale of variation of ge-

32 nome size, encompassing a 100,000-fold range across eukaryotes (Gregory 2021).

33 Decades of research have revealed the consistent covariation of two organismal fea-

34 tures with genome size: a negative correlation with cell division rate, and a positive cor-

35 relation with cell size (Gregory 2001, 2005). Genome size has also been associated

36 with a variety of organismal or ecological factors including: developmental rate or com-

37 plexity (Gregory 2002b), temperature (Hessen, et al. 2013), metabolic rate (Waltari and

38 Edwards 2002; Roddy, et al. 2019), invasiveness (Pandit, et al. 2014), or speciation and

39 extinction rates (Vinogradov 2004; Jeffery, et al. 2016), but these associations vary

40 across studies.

41 Comparative biologists often think of adaptive explanations for character associa-

42 tions, which would suggest that genomes evolve toward an "optimum" size with respect

43 to one or more of these correlated traits. However, the context-sensitive nature of asso-

44 ciations with these factors belies a strongly adaptive explanation for genome size. For

45 example, metabolic rate and genome size are correlated in some vertebrate clades, but

46 not in others, and genome size provides no overall explanatory power for basal meta-

47 bolic rate across vertebrates (Licht and Lowcock 1991; Gregory 2002a; Smith, et al.

48 2013; Uyeda, et al. 2017; Gardner, et al. 2020). Genome size evolution may instead

49 evolve nearly neutrally until some threshold value is reached, beyond which fitness is 
50 impacted (Gregory 2002b). This process is more aptly described as governed by con-

51 straints. As far as we are aware, the constraint model has never been formally tested

52 within a phylogenetic comparative framework for a univariate trait.

53 Recent years have seen tremendous improvement in our understanding of the

54 mechanistic processes by which genome size evolves. Variation in genome size can re-

55 flect the accumulation of many types of sequences, from simple repeats to increases in

56 ploidy (Elliott and Gregory 2015; Pasquesi, et al. 2018; Carta, et al. 2020), such that the

57 majority of the genome is non-coding or "junk" DNA. In vertebrate animals, genome size

58 is strongly determined by the accumulation of transposable elements (TEs), sequences

59 that replicate and spread throughout host genomes (Sotero-Caio, et al. 2017; Shao, et

60 al. 2019). TEs are also deleted by mutations introduced during replication, recombina-

61 tion, and DNA repair (Michael 2014; Vu, et al. 2017). In the absence of selection or con-

62 straint on genome size, the background process for genome evolution is stochastic, with

63 genome size increasing if TE insertions outpace deletions. TE activity is often neutral at

64 the cellular and organismal levels, with most individual insertions and deletions missing

65 functional regions of the genome and resulting in negligible fitness consequences

66 (Arkhipova 2018). Non-coding DNA can therefore accumulate until genome size

67 crosses a threshold where it begins to impact fitness through a correlated trait (e.g. cell

68 size or developmental rate).

While the notion of constraint on genome size is conceptually appealing, there

70 are few comparative methods that can detect constraint and possibly distinguish it from

71 adaptation. Whether by correlation of phylogenetically independent contrasts 
72 (Felsenstein 1985) or by phylogenetic autocorrelation (Cheverud, et al. 1985), phyloge-

73 netic comparative methods that focus primarily on the mean or "location" of the pheno-

74 type are primed to detect adaptive evolution, as selection is expected to move the phe-

75 notype toward an optimum (Simpson 1953; Lande 1980). For example, testing for a cor-

76 relation between traits after independent contrasts (Felsenstein 1985) may reveal

77 whether, on average, a phenotype covaries across species with some other trait, with

78 the relationship maintained presumably as a result of adaptive evolution. Phylogenetic

79 autocorrelation (Cheverud, et al. 1985) and phylogenetic regression (Grafen and

80 Hamilton 1989) use regression approaches to separate phylogenetic and environmental

81 (adaptive) effects on variation of the mean phenotype. Ancestral character state recon-

82 struction methods can infer the mean phenotype of interest along nodes of the tree and

83 are used to explore evolution in relation to some other factor, but vary in the underlying

84 models employed (Huey and Bennett 1987; Maddison 1991; Schluter, et al. 1997). How

85 mean-focused methods enable the detection of constraint, however, is unclear. Meth-

86 ods that model both the mean and variance of a stochastically evolving phenotype, such

87 as Ornstein-Uhlenbeck-based methods (Hansen 1997; Butler and King 2004; O'Meara,

88 et al. 2006; Beaulieu, et al. 2012), may open the door to exploring constraint by reveal-

89 ing whether a stochastically spreading phenotype evolves by selection, or by weak se-

90 lection bounding the phenotype consistent with the presence of constraint, or by no se-

91 lection at all.

92 Salamanders are an ideal group to test hypotheses of selection and constraint on

93 genome size. They have both the largest genomes and the largest range of variation in

94 genome size among vertebrates. Genome sizes range from $9 \mathrm{~Gb}-120 \mathrm{~Gb}$ across the 
95763 extant species (Decena-Segarra, et al. 2020; AmphibiaWeb 2021; Gregory 2021),

96 reflecting varying levels of TE accumulation. Salamanders also have exceptional life-

97 history diversity, in particular, with metamorphosis lost, modified, and regained through-

98 out their evolutionary history (Wiens, et al. 2005; Bonett, et al. 2014). Metamorphosis

99 has been hypothesized to shape genome size evolution because of the effects that ge-

100 nome (and therefore cell) size has on the rate of development (Wake and Marks 1993;

101 Gregory 2002b). Because metamorphosis can be a vulnerable part of the life cycle, its

102 duration has been proposed as a target of natural selection. Selection to limit the time in

103 metamorphosis would indirectly select for faster development, constraining genomes

104 (and cells) to smaller sizes (Gregory 2002b; Bonett, et al. 2020).

105 Several studies have linked life history to genome size in salamanders, with

106 smaller (albeit still enormous relative to other taxa) genome sizes associated with meta-

107 morphosis (Wake and Marks 1993; Gregory 2002b; Sessions 2008; Bonett, et al. 2020).

108 Bonnett (2020) used phylogenetic comparative methods to demonstrate that salaman-

109 der genome size is better explained by models that account for differences in life history

110 - including the presence or absence of metamorphosis - as opposed to variation in

111 ecology or habitat stability. However, this study was largely focused on the effects of

112 facultative loss of metamorphosis on genome size evolution; thus, it did not explore the

113 concept of evolutionary constraint versus adaptation. In addition, it did not examine the

114 connection between energetic vulnerability and metamorphic duration, which we pro-

115 pose as a key conceptual link between metamorphosis and genome size. 
In this study, we build on this previous body of work, revisiting the hypothesis that

117 the radical morphological repatterning associated with metamorphosis imposes evolu-

118 tionary constraints on genome size in salamanders. In particular, we model characteris-

119 tics that are likely to place energetic limits on the duration of metamorphosis: Does re-

120 patterning happen inside the egg, fueled solely by yolk stores? Does repatterning hap-

121 pen in an organism that is free-living, but unable to feed? We use life history and ge-

122 nome size data to inform stochastic models of trait evolution that explore how different

123 metamorphic regimes interact with genome expansion. More generally, we demonstrate

124 the ability of OU-based stochastic models to identify trait evolution governed by con-

125 straint, expanding their use beyond classic scenarios of adaptive evolution.

127 Life History Regimes Constraining Genome Size Evolution

129 In the absence of any selection or constraint, genome size in salamanders is expected

130 to expand by biased stochastic evolution. Overall mutation pressure in the clade leads

131 to TE accumulation, as TE deletion rates are low and TE silencing is incomplete (Sun,

132 Arriaza, et al. 2012; Frahry, et al. 2015; Madison-Villar, et al. 2016; Mohlhenrich and

133 Mueller 2016). Salamanders' enormous genomes are the cumulative result of unusually

134 high levels of TE activity and retention (Sun, Shepard, et al. 2012; Sun and Mueller

135 2014; Keinath, et al. 2015; Liedtke, et al. 2018; Nowoshilow, et al. 2018).

The ancestral salamander life history included a larval growth stage followed by

137 metamorphosis. Metamorphosis is a radical transformation of morphology during the

138 life cycle that produces strikingly different, largely decoupled larval and adult forms 
139 (Moran 1994). During metamorphosis, as during embryogenesis, salamanders undergo 140 rapid cell division, differentiation, migration, and apoptosis (Alberch 1989). The dynam-

141 ics of these cellular processes are affected by genome size and cell size; as ge-

142 nome/cell sizes increase, developmental rates throughout ontogeny — from embryo-

143 genesis through metamorphosis — slow down (Horner and Macgregor 1983; Jockusch

144 1997). Metamorphosis has been lost and regained from the life cycle numerous times

145 by different mechanisms (Chippindale, et al. 2004; Mueller, et al. 2004; Wiens, et al.

146 2005; Bonett, et al. 2014), producing multiple life history regimes that could shape ge-

147 nome size evolution. We outline these regimes, and their predicted effects on genome

148 size evolution, below and in Figure 1:

149 Paedomorphosis: In paedomorphic species, some or all stages of metamorphic repat-

150 terning are lost, and organisms reach sexual maturity retaining largely larval traits

151 (Gould 1977). Because there is no selection to limit time in metamorphosis, there is no

152 associated constraint on genome size. Thus, paedomorphs are expected to most

153 closely reflect the background condition of stochastic genome expansion.

154 Direct development: In direct-developing species, the larval growth stage is eliminated,

155 and embryogenesis and metamorphosis are integrated into a single sequence of devel-

156 opmental events that takes place inside the egg (Alberch 1989; Rose 2014). This entire

157 sequence must be fueled by yolk provisioned by the mother. Thus, we might expect se-

158 lection to limit time in metamorphosis, imposing a constraint on genome size.

159 Non-feeding metamorphosis: Within the salamander family Plethodontidae, metamor-

160 phic repatterning events happen relatively synchronously in a free-living organism. Dur-

161 ing this metamorphosis, the organisms are unable to feed because of the replacement 
162 of cartilaginous elements associated with the change from suction to projectile feeding

163 (Rose 1995a; Deban and Marks 2002). The entire process must be fueled by energy re-

164 serves built up during the larval stage. Thus, we might expect selection to limit time in

165 metamorphosis, imposing a constraint on genome size.

166 Feeding metamorphosis: In non-plethodontid salamanders, metamorphic repatterning

167 events happen in a free-living organism that is able to feed throughout the transfor-

168 mation. Thus, we would not expect selection based on energy demands to limit time

169 spent in metamorphosis. There are, however, other ways in which metamorphosis can

170 increase vulnerability that might translate into selection on metamorphic duration, con-

171 straining genome size. 
bioRxiv preprint doi: https://doi.org/10.1101/2021.05.05.442795; this version posted May 6,2021 . The copyright holder for this preprint (which was not certified by peer review) is the author/funder, who has granted bioRxiv a license to display the preprint in perpetuity. It is made available under aCC-BY-NC-ND 4.0 International license.
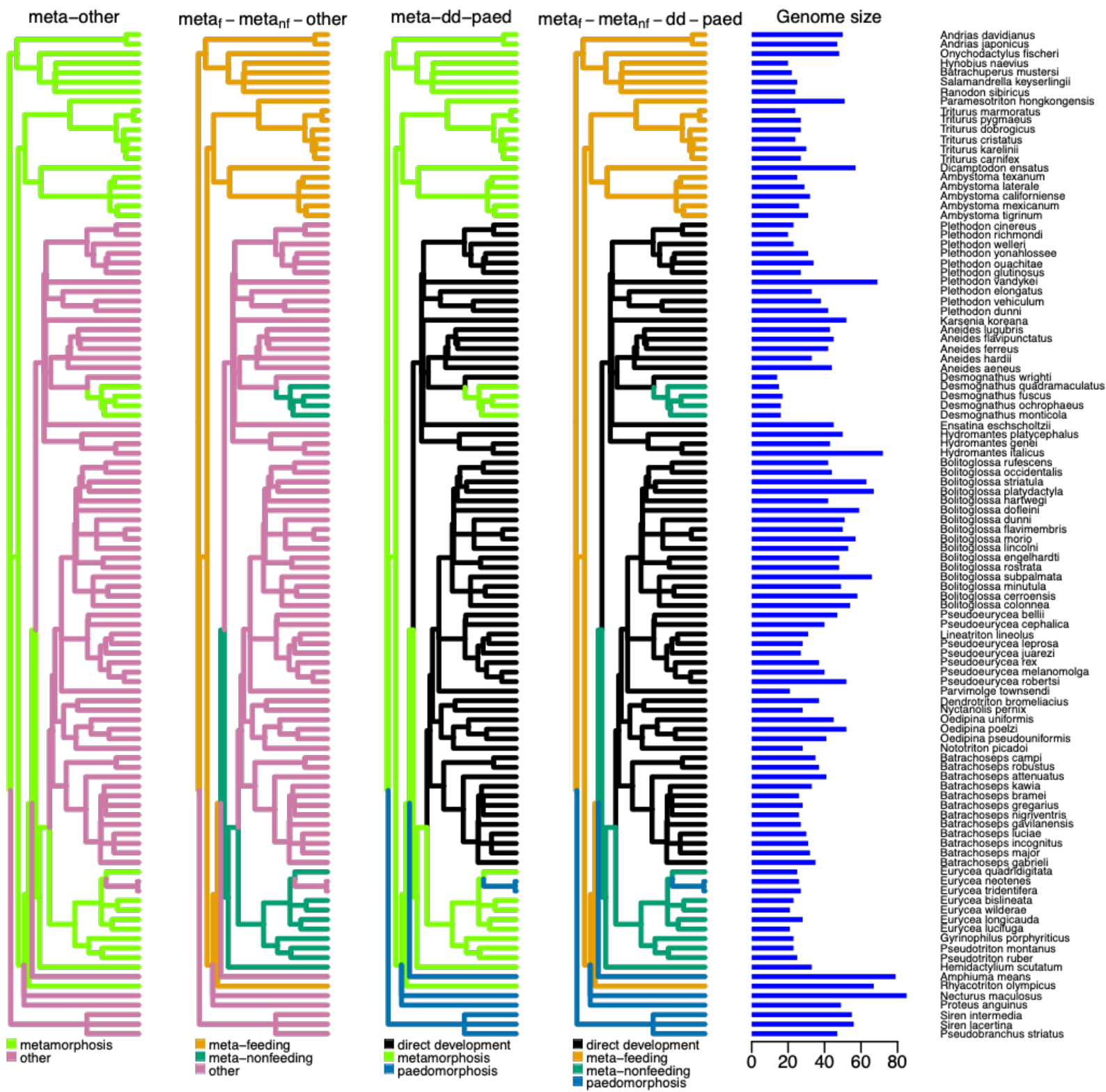

174 Figure 1. Alternative hypotheses for constraints imposed by development on genome size evolution in salamanders. On each phylogeny, alternative life history regimes are painted in different colors as indicated in each legend (see text). Genome sizes are shown on the right in $\mathrm{pg}(1 \mathrm{pg}=978 \mathrm{Mb})$. 


\section{Methods}

Taxon Sampling, Genome Size, and Phylogeny

184 Genome size data were available for 106 species of salamanders (out of a total of 763

185 currently named extant species), encompassing all ten salamander families and 35 of

18668 genera (Supplemental Table 1) (AmphibiaWeb 2021; Gregory 2021). We trans-

187 formed the data with natural logarithms prior to analysis. Our sampling includes repre188 sentatives with diverse life histories: direct development, paedomorphosis, feeding met-

189 amorphosis, and non-feeding metamorphosis. Hereafter, we distinguish between "feed-

190 ing" and "non-feeding metamorphosis", and use "metamorphosis" to indicate all species

191 which undergo metamorphosis, whether feeding or non-feeding. Several lineages are

192 facultative paedomorphs, which retain the ability to undergo metamorphosis under cer-

193 tain conditions. We coded these taxa as metamorphic, reflecting the experience of con-

194 straint, even if only occasionally, and supported by recent work examining the evolution-

195 ary impacts of facultative paedomorphosis (Bonett, et al. 2020).

We used a previously published phylogeny (Mueller, et al. 2008; Pyron and

198 Wiens 2011; Vieites, et al. 2011; Zheng, et al. 2011) and estimated branch lengths on

199 this topology using sequence data for two mitochondrial genes - cytochrome-b and 16S

200 obtained from GenBank. Additional Sanger sequences were collected as necessary at

201 the Colorado State University sequencing core facility or the Joint Genome Institute fol-

202 lowing (Mueller, et al. 2004) (Supplemental Table 1). Loci were aligned using MUSCLE 
203 (Edgar 2004). Branch lengths were estimated for each locus independently and then av-

204 eraged, weighted by gene length. Branch lengths were estimated using RAxML

205 (Stamatakis 2006) using the best-fit (AIC) model of nucleotide substitution in ModelTest

2063.6 (Posada and Crandall 1998), with Cyt-b partitioned by codon. The resulting tree was

207 ultrametricized using penalized likelihood implemented in r8s with the truncated Newton

208 algorithm and cross validation to select the optimal smoothing parameter value

209 (Sanderson 2003).

210

211 Models of Genome Size Evolution

213 We modeled genome size evolution using both Brownian motion (BM) and Ornstein-Uh-

214 lenbeck (OU) models of evolution (Hansen 1997; Butler and King 2004; O'Meara, et al.

215 2006; Beaulieu, et al. 2012). As these models have been described previously, we

216 cover them only briefly here. The BM model is the simplest stochastic model used in

217 comparative analysis and has a single rate parameter $\sigma$, which can be thought of as a

218 stochastic noise intensity parameter shared by all taxa and describing the magnitude of

219 the independent random walks of the trait evolving along the branches of the phylogeny.

220 This model predicts that across lineages, there will be no change in mean phenotype,

221 but variance will grow through time as lineages wander over trait space, and the ex-

222 pected variance between two lineages will be proportional to the time since their diver-

223 gence. Conceptually, the BM model may be a good candidate for purely stochastic ge-

224 nome size evolution if increases occur as frequently as decreases. 
The multiple-rate BM model, introduced by O'Meara, et al. (2006), allows different $\sigma$ values across different portions of a phylogenetic tree. We use this model to rep-

227 resent changes in the rate of stochastic evolution accompanying changes in constraint 228 resulting from shifts in life history regime. Under this model, lineages evolving under dif229 ferent regimes may differ in variance.

232 shift as well as allowing variance to narrow. They include a deterministic component of

233 trait evolution that models the tendency of the trait to move toward an equilibrium value.

234 Mathematically, the model for trait evolution expressed as a differential equation is

$$
d X(t)=\alpha(\theta(t)-X(t))+\sigma d B(t)
$$

where $\theta(t)$ is the deterministic equilibrium for the trait at time $t$ and $\alpha$ is an evolutionary rate describing the strength of the deterministic pull (e.g. selection) towards that equilib-

240 rium along a branch of a tree. Hypotheses regarding trait evolution are specified by

241 painting "regimes" on the tree to indicate where these parameters are expected to shift.

242 The simplest OU models allow multiple equilibria leading to the evolution of differences

243 in mean phenotype across regimes (Hansen 1997; Butler and King 2004); for example,

244 in the current study, separate equilibria might exist for paedomorphs, metamorphosers,

245 and direct developers. Further model extensions also allow the strength of the determin-

246 istic pull and the stochastic noise intensity to vary across regimes (Beaulieu, et al. 
247 2012), as might be consistent with the lifting or imposition of constraints on genome size 248 evolution.

We formalized five hypotheses for how life history regime could constrain ge-

250 nome size evolution in salamanders: (1) Brownian motion: Genome size evolves by

251 purely stochastic evolutionary processes with neither constraint nor bias. (2) metamor-

252 phosis-other: Metamorphosis imposes a constraint on genome expansion that is dis-

253 tinct from the other life histories. (3) meta-paed-dd: Metamorphosis, direct develop-

254 ment, and paedomorphosis each impose distinct constraints on genome expansion. (4)

255 meta $_{f}-$ meta $_{n f}$-other: Feeding metamorphosis (meta $)$ and non-feeding metamorphosis

$256\left(\right.$ meta $\left._{n f}\right)$ each impose constraints that are distinct from those experienced by salaman-

257 der species with other life histories. (5) meta-meta mf $_{f}$-paed-dd: non-feeding metamor-

258 phic species, feeding metamorphic species, paedomorphic species, and direct-devel-

259 oping species each experience different levels of constraint on genome expansion.

$260 \quad$ These five biologically-inspired hypotheses are specified by fitting evolutionary

261 models that allow their parameters to vary with life history regime. The simplest form al-

262 lows the equilibrium to vary with life history regime while assuming the rates of evolution

263 are constant across the phylogeny. Additional subhypotheses fit evolutionary models

264 that vary stochastic noise intensity values $(\sigma)$, and/or deterministic pull values $(\alpha)$ ac-

265 cording to life history regime (Table 1). In all, we tested 21 models to explore life history

266 constraints on genome size and model parameters (Table 2). We fit all of the models

267 with the character state of the node at the base of the plethodontid lineage defined as

268 metamorphosing as well as direct-developing (Bonett, et al. 2014), and found that the

269 choice of the basal character state made no qualitative difference to our conclusions. In 
270 addition, we made a series of pairwise comparisons to further assess the degree of in-

271 fluence of feeding metamorphosis, paedomorphosis, direct development, and non-feed-

272 ing metamorphosis on genome size evolution in combinations of increasing complexity

273 (Table 3).

274 We fit models of evolution using the software package OUwie (Beaulieu, et al.

275 2012) and compared the multiple optimum OU models with results fitted in OUCH

276 (Butler and King 2004; King and Butler 2009). We modified OUwie to fit models with re-

277 gime changes that occur at the nodes, as in $\mathrm{OUCH}$, rather than the default behavior of

278 OUwie which places regime changes mid-way along the branch between a clade of in-

279 terest and its ancestor. We recognize that while either choice can be argued to be arbi-

280 trary, assuming that the regime change occurred simultaneously with the origin of the

281 clade that bears the phenotype as indicated in Figure 1 seems reasonable. All analyses

282 were conducted in the R statistical computing environment (R Core Team 2020). We

283 note here that OUwie has different options for dealing with the root state, $X(0)$. Absent

284 any information about the phenotype deep in the tree, this parameter is often very diffi-

285 cult to estimate in OU models (Cressler, et al. 2015). One alternative is to assume that

286 the value of $X(0)$ is distributed according to the stationary distribution of an OU process,

287 which eliminates this parameter by absorbing the variance into the covariance matrix

288 implied by the phylogeny itself (Ho and Ané 2013). However, OUwie does not currently

289 support this approach for OU models with multiple $\alpha$ or $\sigma$ parameters, and as far as we

290 know, the mathematical modifications for these models have not been worked out. Be-

291 cause the option to estimate the value of the root node as in the earlier implementations 
292 (Hansen 1997; Butler and King 2004); and specified in OUwie by setting root.sta-

293 tion=FALSE) is available for all of the models of interest, we used it for all model fitting.

294 However, as we show in the Supplementary Materials, the parameter estimates of a sin-

295 gle- $\alpha$, single- $\sigma$ OU model fit assuming the root is in the stationary distribution are very

296 different from the parameter estimates obtained by fitting the root state, although model

297 selection conclusions hold. This large difference in the parameter values reinforces a

298 general point in phylogenetic comparative hypothesis testing, which is that parameter

299 estimation is often more fraught than hypothesis testing, and as such, parameter esti-

300 mates should be interpreted with caution (Beaulieu, et al. 2012; Ho and Ané 2013; Ho

301 and Ané 2014; Cressler, et al. 2015; Cooper, et al. 2016).

When fitting OU models, there are datasets for which it is possible to recover an

303 extremely low value of $\alpha$ (weak deterministic pull), with equally extremely large $\theta$ (equi-

304 librium values). This combination can reasonably be interpreted as weak selection, or a

305 biased nearly-random walk. Rather than look only at these parameters in isolation, we

306 considered whether it may be more informative to determine their combined effect on

307 trait evolution. To this end, we calculated the value of $\alpha(X-\theta)$ for each life history re-

308 gime to estimate any "deterministic trend" imposed by the best-fitting model, where $X$ is

309 the average genome size of species in that regime at the end of the evolutionary pro-

310 cess. 
311 Table 1. BM and OU models with single or multiple parameters used to fit the data.

312 Numbers in parentheses specify (1) model parameters and notation, (2) parameters that

313 remain constant across the phylogeny, (3) parameters that vary with shifts in life history

314 regime, (4) O'Meara (2006) model notation, and (5) notes for the model implementa-

315 tions and citations.

316

\begin{tabular}{|c|c|c|c|c|}
\hline Models & Uniform & $\begin{array}{c}\text { Variable } \\
\text { with Re- } \\
\text { gime }\end{array}$ & $\begin{array}{l}\text { O'Meara } \\
\text { Notation }\end{array}$ & Notes \\
\hline (1) & (2) & (3) & (4) & (5) \\
\hline Single noise intensity $\quad \sigma$ & $\sigma$ & & BM1 & $\begin{array}{l}\text { Classic BM model of } \\
\text { (Felsenstein 1985) }\end{array}$ \\
\hline Multiple noise intensities $\sigma_{i}$ & & $\overline{\sigma_{i}}$ & BMS & $\begin{array}{l}\text { Multiple-rate BM model of } \\
\text { O'Meara et al. (2006) }\end{array}$ \\
\hline Multiple equilibria $\quad \theta i, \sigma, \alpha$ & $\sigma$ & $\theta_{i}$ & OUM & $\begin{array}{l}\text { OU model of Butler and } \\
\text { King (2004) }\end{array}$ \\
\hline $\begin{array}{l}\text { Multiple equilibria and determinis- } \\
\text { tic pull strengths } \theta i, \sigma, \alpha i\end{array}$ & $\sigma$ & $\begin{array}{l}\alpha_{i} \\
\theta_{\iota}\end{array}$ & OUMA & $\begin{array}{l}\text { Multiple- } \alpha \text { model of Beau- } \\
\text { lieu et al. (2012). }\end{array}$ \\
\hline $\begin{array}{l}\text { Multiple equilibria and noise in- } \\
\text { tensities } \theta i, \sigma i, \alpha\end{array}$ & $\alpha$ & $\begin{array}{l}\sigma_{i} \\
\theta_{i}\end{array}$ & OUMV & $\begin{array}{l}\text { Multiple- } \sigma \text { model of Beau- } \\
\text { lieu et al. (2012) }\end{array}$ \\
\hline
\end{tabular}


bioRxiv preprint doi: https://doi.org/10.1101/2021.05.05.442795; this version posted May 6, 2021. The copyright holder for this preprint (which was not certified by peer review) is the author/funder, who has granted bioRxiv a license to display the preprint in perpetuity. It is made available under aCC-BY-NC-ND 4.0 International license.

\begin{tabular}{|l|l|l|l|l|}
\hline Multiple equilibria, noise intensi- & & $\sigma_{i}$ & OUMVA & Full model of Beaulieu et \\
ties, and deterministic pull & & $\alpha_{i}$ & & al. (2012). \\
strengths $\theta i, \sigma i, \alpha i$ & & $\theta_{i}$ & & \\
\hline
\end{tabular}




\section{Model Comparison and Parameter Estimation}

320 We compared the fit of each of the models using the Akaike Information Criterion cor-

321 rected for small sample size $\left(\mathrm{AIC}_{\mathrm{c}}\right)$. We further performed model selection bootstrap

322 analysis (phylogenetic Monte Carlo; (Boettiger, et al. 2012) for targeted comparisons of

323 the models in Table 3 to assess the strength of evidence for different hypotheses as

324 well as the power to detect differences in model support. Model selection bootstrap

325 analysis is necessary because $\mathrm{AIC}_{\mathrm{c}}$ differences can favor more complicated models,

326 even when a simpler model is correct (Boettiger, et al. 2012). For each comparison, we

327 computed the observed likelihood difference,

$$
\delta_{\text {obs }}=-2\left(\log L_{0}-\log L_{1}\right)
$$

where $L_{0}$ is the likelihood of the simpler model and $L_{1}$ is the likelihood of the more complex model.

Determining whether $\delta_{\text {obs }}$ is significantly different from a null expectation requires an approximate $p$-value — the probability of observing $\delta_{\text {obs }}$ if the simpler model were

333 true. That is, we need to compare the value $\delta_{\text {obs }}$ to the distribution of $\delta$ values under the

334 simpler model. To create this distribution, we generated 500 datasets by simulating the

335 simpler model at its MLE parameter estimates; we then fit both the simpler and more

336 complex models to each simulated dataset and computed the values of $\delta$, producing a

337 null distribution of $\delta$ under the simpler model. We compared the observed value of $\delta$ to

338 this null distribution to calculate an approximate $p$-value.

340 more complex model is true. To estimate power, we generated 500 datasets by simulat-

341 ing the more complex model at its MLE parameter estimates; we then fit the two models 
342 and computed the values of $\delta$. The fraction of these $\delta$ values that are greater than the

$34395 \%$ quantile of the distribution generated under the simpler model (described above)

344 gives an estimate of power. All data and code necessary to carry out the analysis in this

345 manuscript can be found at https://github.com/claycressler/genomesize and in Supple-

346 mental Material.

$348 \quad$ Results

The best-fitting model for salamander genome size evolution accounted for four

351 regimes: both non-feeding and feeding metamorphosis, paedomorphosis, and direct de-

352 velopment $\left(\right.$ meta $_{f}-$ meta $_{n f}-$ paed-dd; Table 2) under an OU model that allowed both equi-

353 librium genome size and noise intensity to vary across these regimes $\left(\theta_{\mathrm{i}}, \sigma_{\mathrm{i}}, \alpha\right.$, Table 2$)$.

354 An identical 4-regime model with only a single noise intensity fit nearly as well (Table 2).

355 Additionally, the three-regime meta $_{-}-$meta $_{n f}$-other $\left(\theta_{\mathrm{i}}, \sigma_{\mathrm{i}}, \alpha\right)$ hypothesis provided some

356 explanatory power. These three models were far superior to the remaining models.

357 Overall, the addition of multiple $\alpha$ values resulted in worse model fit relative to a

358 uniform $\alpha$ value, whereas the addition of multiple $\sigma$ values relative to a single $\sigma$ both im-

359 proved and worsened model fit, depending on the hypothesis (Table 2). We present the

360 results with the character state of the node at the base of the plethodontid lineage de-

361 fined as metamorphosing. In the Supplementary Materials, we show that defining this

362 node as direct-developing has no effect on the evolutionary conclusions we draw here. 
The model selection bootstrap analysis allows us to quantify the degree of improvement in explanatory power provided by moving between specific hypotheses (Figure 2). Note that for each comparison, we used the version of the OU model that had

366 the lowest AICc (Table 2), so comparisons involving metamorphosis-other and meta-

367 paed-dd used the single- $\sigma$ model, whereas comparisons involving meta $_{f}-m_{\text {eta }}$-paed-

$368 d d$ and meta $_{f}-$ meta $_{n f}$-other used the multiple- $\sigma$ model.

Based on these results, we can reject any purely stochastic hypothesis for genome size evolution, as a model that allows for separate equilibrium values for meta-

371 morphosers was far superior to any purely neutral model (Table 2; Figure 2A). Specify-

372 ing distinct equilibrium values for non-feeding and feeding metamorphosis substantially

373 improves the explanatory power of any model (Figure 2C, D). In particular, the separa-

374 tion of metamorphosis into feeding and non-feeding categories provided far greater im-

375 provement of the model than subdividing the "other" category into direct development

376 and paedomorphosis (compare the results in Figure 2C to those in Figure 2B, and Fig-

377 ure 2D to $2 \mathrm{E}$ ). Allowing distinct noise parameters for each regime slightly improves the

378 fit of the OU models with separate equilibria for non-feeding and feeding metamorphosis

379 (Table 2), although the improvement over the single- $\sigma$ model is not significant (Compari-

380 son [6], Table 3; Figure 2F). Therefore, the best model included distinct equilibrium val-

381 ues for direct development, paedomorphosis, feeding metamorphosis, and non-feeding 382 metamorphosis. 
Table 2. Model comparison statistics. Best models (interrogated by bootstrap, Table 3) indicated in bold. Model parameterizations are indicated by: $\sigma=$ Brownian motion; $\sigma_{i}=$ Brownian motion with multiple noise intensities; $\theta_{i}, \sigma, \alpha=$ OU model with multiple equilibria; $\theta_{i}, \sigma_{i}, \alpha=\mathrm{OU}$ model with multiple equilibria and multiple noise intensities; $\theta_{i}, \sigma, \alpha_{i}$ $=$ OU model with multiple equilibria and multiple deterministic pull strengths; $\theta_{i}, \sigma_{i}, \alpha_{i}=$ OU model with multiple equilibria, noise intensities, and deterministic pull strengths.

\begin{tabular}{|c|c|c|c|c|c|c|}
\hline$\triangle \mathrm{AlC}_{\mathrm{c}}$ & \multicolumn{6}{|c|}{ Model } \\
\hline & \multicolumn{4}{|c|}{ OU Models } & \multicolumn{2}{|c|}{ BM Models } \\
\hline Hypotheses & $\theta i, \sigma i, \alpha$ & $\theta i, \sigma, \alpha$ & $\theta i, \sigma i, \alpha i$ & $\theta i, \sigma, \alpha i$ & $\sigma i$ & $\sigma$ \\
\hline $\begin{array}{c}\text { meta }_{f}-\text { meta }_{n f}-\text { paed- } \\
d d\end{array}$ & 0 & $1.1^{a}$ & 7.5 & 8.1 & 12.9 & \\
\hline meta $_{f}-$ meta $_{n f}-$ other & $1.4^{b}$ & 3.6 & 6.1 & 8.1 & 11.1 & \\
\hline meta-paed-dd & 6.0 & 3.4 & 10.7 & 7.9 & 11.4 & \\
\hline metamorphosis-other & 5.8 & 4.1 & 8.0 & 6.3 & 9.5 & \\
\hline Brownian motion & & & & & & 31.8 \\
\hline
\end{tabular}

${ }^{a}$ meta $_{f}-$ meta $_{n f}-d d$-paed with single or multiple noise parameters $\sigma$ are compared in Figure $2 \mathrm{~F}$. 

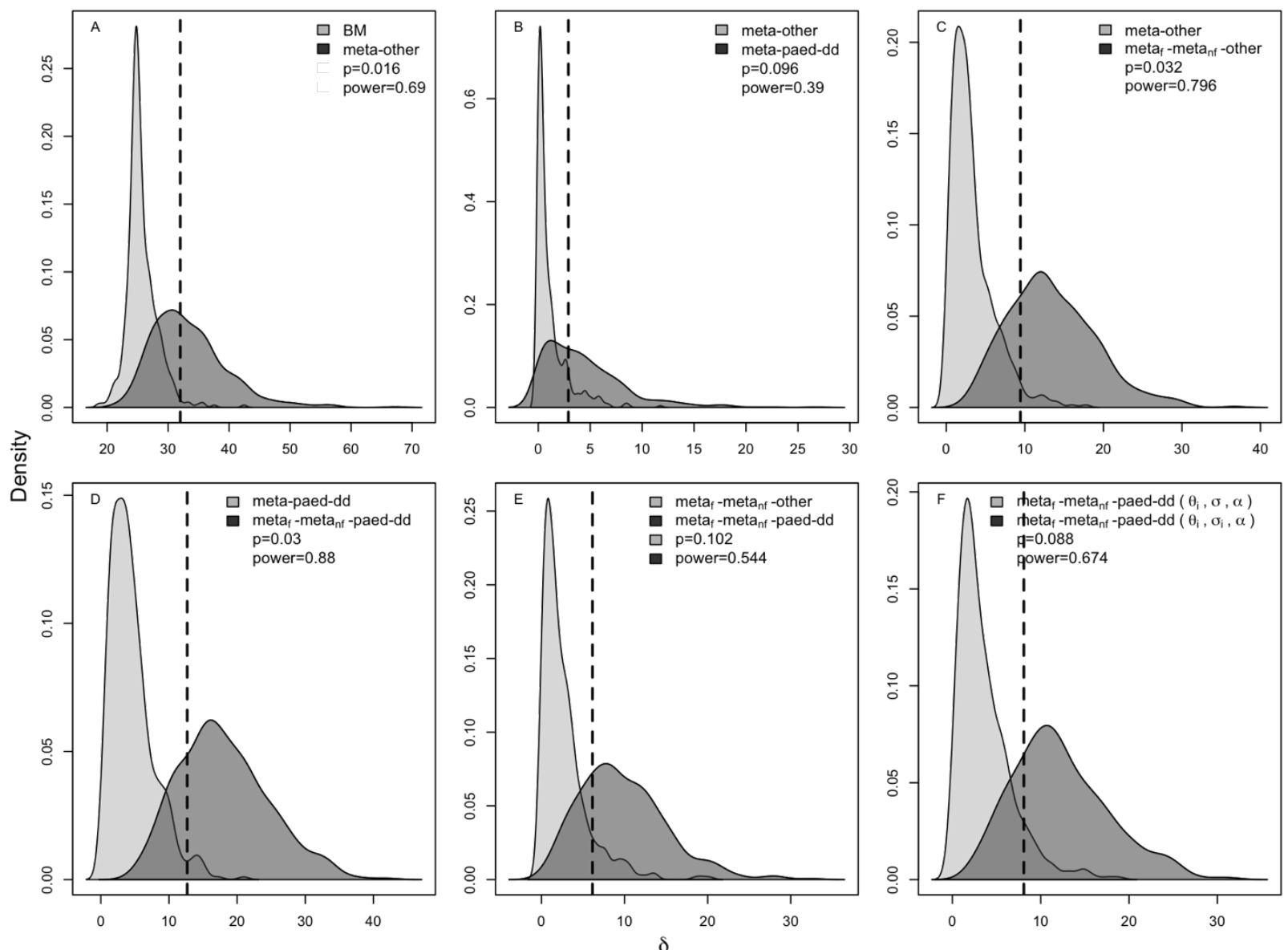

Figure 2. Bootstrap distributions of the likelihood difference $(\delta)$ calculated by generating 500 datasets under each of two competing models at their MLE parameter estimates, refitting the two models, and computing $\delta$. The light gray region shows the probability density of the parameter when the data is generated by the simpler model; the dark gray region shows the density when the data is generated by the more complex model. The dashed line gives the observed value $\left(\delta_{\text {obs }}\right)$ from fitting the actual genome size data. The reported $p$-value is the fraction of the light gray distribution that lies to the right of $\delta_{\text {obs; }}$ the power is the fraction of the dark gray distribution that lies to the right of the 95th percentile of the light gray distribution. Each panel evaluates the support for a different hypothesis: (A) metamorphosis imposes a constraint on genome expansion; (B) there are distinct constraints imposed by the different non-metamorphosing strategies, direct development and paedomorphosis; (C) non-feeding metamorphosis imposes a distinct constraint from feeding metamorphosis; (D) non-feeding metamorphosis imposes a distinct constraint from feeding metamorphosis, after accounting for differences in non-metamorphosing strategies; (E) non-metamorphosing strategies impose unique constraints, after accounting for differences between feeding and non-feeding metamorphosis; (F) after identifying metar-metanf-paed-dd as best-fitting, this comparison tested whether fitting each regime with a distinct stochastic noise intensity improved fit to the data. 
421 Parameter values for each regime estimated under the best-fitting model are presented

422 in Table 3. Direct-developers, non-feeding metamorphosers, paedomorphs, and feeding

423 metamorphosers have broadly overlapping stochastic noise intensity $(\sigma)$ values. Deter-

424 ministic pull strength $(\alpha)$ takes an extremely small value, and the equilibrium values $(\theta)$

425 take extreme values both large and small. Although the deterministic pull strength pa-

426 rameter is vanishingly small, nevertheless, the models that account for metamorphosis

427 are far superior to a purely stochastic model (Table 2 ). We note that the extreme nature

428 of the parameter estimates is a consequence of the handling of the root state (see Sup-

429 plementary Materials).

430

431 
432 Table 3: Maximum likelihood parameter estimates and parametric bootstrap confidence 433 intervals for the best-fitting model (meta -meta $_{n f}-d d$-paed $\theta i, \sigma i, \alpha$ : separate equilibrium 434 values and noise intensities for lineages in the four life history regimes: $d d=$ direct de435 velopment, meta $_{n f}=$ non-feeding metamorphosis, meta $_{f}=$ feeding metamorphosis, paed 436 = paedomorphosis).

437

\begin{tabular}{|c|c|c|c|}
\hline Parameter & & MLE & $95 \% \mathrm{Cl}$ \\
\hline Deterministic pull strength & $\alpha$ & $1.05 \mathrm{e}-7$ & $(1.01 \mathrm{e}-7,1.7)$ \\
\hline \multirow[t]{4}{*}{ Stochastic noise intensity } & $\sigma_{d d}$ & 0.365 & $(0.308,0.545)$ \\
\hline & $\sigma_{m e t a \_n f}$ & 0.355 & $(0.252,0.525)$ \\
\hline & $\sigma_{\text {meta } f}$ & 0.187 & $(0.108,0.283)$ \\
\hline & $\sigma_{\text {paed }}$ & 0.249 & $(0.112,0.464)$ \\
\hline \multirow[t]{4}{*}{ Equilibrium value } & $\theta_{d d}$ & $-3.77 e 5$ & $(-2.67 \mathrm{e} 6,1.99 \mathrm{e} 6)$ \\
\hline & $\theta_{\text {meta_nf }}$ & 3.62 & $(3.32,3.90)$ \\
\hline & $\theta_{\text {meta_f }}$ & $-5.55 e 6$ & $(-7.62 \mathrm{e} 6,3.28)$ \\
\hline & $\theta_{\text {paed }}$ & $4.41 \mathrm{e} 6$ & $(3.96,6.29 \mathrm{e} 6)$ \\
\hline
\end{tabular}


440 There is evidence of a deterministic trend $\alpha(X-\theta)$ towards small genome size in sal-

441 amanders that undergo non-feeding metamorphosis as well as a trend towards large

442 genome size in paedomorphic salamanders (Table 4). In contrast, genome sizes in di-

443 rect developing and feeding metamorphosing salamanders show no such trends. The

444 range of values for the strength of the overall deterministic trend (Table 4) is much

445 greater than the range of values for stochastic noise intensity (Table 3).

447 Table 4: Estimates of the deterministic trend in the best-fitting model for lineages evolv448 ing in each life history regime, based on the average genome size (log-scale) of sala449 manders in each regime. Genome sizes (in pg) are also shown in parentheses.

\begin{tabular}{|l|l|l|}
\hline \multicolumn{1}{|c|}{ Regime } & \multicolumn{1}{|c|}{ Average log } & Deterministic trend \\
& genome size $X$ & $\alpha(\theta-X)$ \\
\hline Direct development & $3.66(38.9 \mathrm{pg})$ & -0.0396 \\
\hline Feeding metamorphosis & $3.46(31.8 \mathrm{pg})$ & $1.68 \mathrm{e}-8$ \\
\hline Non-feeding metamorphosis & $3.07(21.5 \mathrm{pg})$ & -0.583 \\
\hline Paedomorphosis & $3.89(48.9 \mathrm{pg})$ & 0.463 \\
\hline
\end{tabular}




\section{Discussion}

Biased stochastic expansion and life history constraints shape genome size.-

456 Overall, evolution of genome size in salamanders is well-described by a weakly deter-

457 ministic model with separate equilibria for each life history regime and moderate sto-

458 chasticity. An increased level of constraint in lineages that evolved non-feeding meta-

459 morphosis, as well as a decreased level of constraint in lineages that have lost meta-

460 morphosis, exert the greatest deterministic influences on the evolution of genome size.

461 The strength of the deterministic trend towards genome expansion for paedomorphs is

462 roughly the same as the strength of the deterministic trend towards genome reduction

463 for non-feeding metamorphosers; both are an order of magnitude stronger than the

464 trend for direct-developers. Feeding metamorphosers have no deterministic trend (Ta-

465 ble 4). Taken together, these results, and the observed spread of genome sizes within

466 regimes, suggest that genome size evolution is driven by stochasticity with a bias to-

467 ward increase - likely representing TE insertions that outpace deletions - constrained

468 to varying degrees by metamorphic repatterning, when selection acts to limit metamor-

469 phic duration.

471 Metamorphosis as a vulnerable stage of the life cycle in amphibians

472 Metamorphosis has been posited to exert evolutionary pressure for reduced genome

473 size in amphibians to speed development during a vulnerable life stage. This hypothesis

474 comes primarily from evidence in frogs. Metamorphosing frogs experience higher pre-

475 dation levels because they can neither swim nor hop effectively (Wassersug and Sperry 
1977; Arnold and Wassersug 1978). In addition, frogs are unable to feed during metamorphosis, at a time when their energetic requirements can nearly double as compared

478 to the period immediately preceding metamorphosis (Orlofske and Hopkins 2009;

479 Wright, et al. 2011). Despite being homologous and retaining broad similarities at the

480 transcriptomic, hormonal, and organismal levels (Sanchez, et al. 2018), salamander

481 metamorphosis is different — and less dramatic — than frog metamorphosis. The pro-

482 cess takes much longer in salamanders; timescales are on the order of weeks to

483 months rather than days (Norman 1985; Downie, et al. 2004; Vladimirova, et al. 2012;

484 Sanchez, et al. 2018), suggesting little, if any, time pressure. Metamorphosing salaman-

485 ders do not experience compromised locomotion and are thus not more vulnerable to 486 predation as are frogs (Landberg and Azizi 2010). In addition, metamorphosing sala-

487 manders do not have higher energetic requirements compared to non-metamorphosing

488 individuals of the same species (Vladimirova, et al. 2012). Some salamanders, how-

489 ever, are unable to feed during metamorphosis, requiring that they undergo the transfor-

490 mation using only stored energy reserves (i.e., non-feeding metamorphosers) (Deban

491 and Marks 2002)). Direct developers undergo the transformation inside the egg, fueled

492 only by yolk stores. Genome size constraints for these two life history regimes likely re-

493 flect energetic vulnerability that is not relevant for the other two life histories considered

494 here: feeding metamorphosis and paedomorphosis.

496 Genome size evolution in feeding metamorphosers._Lineages that undergo metamor497 phosis, but are able to feed throughout the process, show no deterministic trend in ge498 nome size evolution. Rather, trait evolution is described by moderate stochastic noise 
499 around an equilibrium value that we interpret as a balance between TE accumulation

500 and a constraint imposed by metamorphosis. Although the animals are able to feed,

501 there are other ways in which fitness can be lowered during salamander metamorpho-

502 sis; for example, metamorphosing individuals are less able to exploit stream habitat re-

503 fugia than either larvae or adults, which increases their mortality (Lowe, et al. 2019).

504 Our results indicate that feeding metamorphosis imposes a less severe constraint on

505 genome size than non-feeding metamorphosis, and we infer that the constraint is medi-

506 ated by vulnerabilities other than depletion of energetic stores.

508 Genome size evolution in non-feeding metamorphosers. - Although other analyses

509 have demonstrated a link between metamorphosis and genome size in salamanders

510 (Wake and Marks 1993; Gregory 2002b; Sessions 2008; Bonett, et al. 2020), here we

511 show that non-feeding metamorphosis imposes a substantial and distinct constraint, rel-

512 ative to feeding metamorphosis, as predicted if energetic vulnerability shapes the dura-

513 tion of metamorphosis. The deterministic trend toward smaller genome sizes within this

514 regime is consistent with the imposition of a more severe constraint against genome ex-

515 pansion — or, put another way, selection towards genome size reduction — to

516 shorten the duration of metamorphosis.

517 The evolution of non-feeding metamorphosis in plethodontids has been an im-

518 portant target of research because the phylogeny suggests that it evolved from a direct-

519 developing ancestor(s), which necessitates the evolutionary reappearance of the "lost"

520 larval stage (Chippindale, et al. 2004; Mueller, et al. 2004). This evolutionary transfor- 
521 mation series was historically considered unlikely, although more recent work has re-

522 vealed potential scenarios for regain of the larval stage (Bonett, et al. 2005; Bonett, et

523 al. 2014). Under this scenario, in the direct-developing ancestral lineage(s), metamor-

524 phic repatterning steps were retained as part of the longer sequence of developmental

525 events that occurred inside the egg (Kerney, et al. 2012). These changes were likely

526 mediated by evolutionary changes in the timing of thyroid hormone activity and re-

527 sponse (Rose 1995a, b; Bonett 2016). The re-evolution of (now non-feeding) metamor-

528 phosis reflected the insertion of a long, slow-growing larval growth phase back into on-

529 togeny, followed by the synchronous occurrence of more drastic metamorphic repattern-

530 ing events in the free-living organism (Rose 1995b, c; Beachy, et al. 2017). Metamor-

531 phic repatterning is more extreme in plethodontids than in other salamanders. Im-

532 portantly, it involves a complete remodeling of the feeding apparatus, with the cerato-

533 branchials (cartilaginous components of the tongue skeleton) replaced by new struc-

534 tures in the adult rather than remodeled from existing larval structures; this results in the

535 inability to feed during metamorphosis (Alberch 1989; Rose 1995c; Deban and Marks

536 2002). Under the classical scenario, in contrast, this synchronization of metamorphosis

537 and drastic remodeling of feeding structures would have evolved in a metamorphosing

538 ancestor to produce non-feeding metamorphosis (Wake and Hanken 2004). Under ei-

539 ther scenario, our results illustrate how phylogenetic comparative methods can reveal

540 the evolutionary forces that have acted on genome size as lineages moved through the

541 different life history regimes. 
543 Genome size evolution in direct developers. - In direct-developing lineages, some or all

544 of the developmental steps of metamorphic repatterning occur inside the egg at the end

545 of embryogenesis (Alberch 1989; Kerney, et al. 2012); these lineages show a weak de-

546 terministic trend towards genome size reduction. Because they are occurring in an em-

547 bryo rather than a free-living organism that has undergone a growth period, the repat-

548 terning happens to a smaller number of cells in a smaller overall mass of tissue com-

549 pared with metamorphosing lineages (Downie, et al. 2004). Thus, the energetic require-

550 ments for comparable developmental steps are lower in direct developers than in meta-

551 morphosers. On the other hand, the energy to fuel these steps comes from yolk stores

552 which, although plentiful in direct developers, are still finite (Wake and Hanken 2004).

553 Thus, we infer that direct development imposes a less severe constraint on genome

554 size than does non-feeding metamorphosis, mediated by the potential for depletion of

555 energy stores if the duration of metamorphic repatterning during embryogenesis is too

556 long. We note that there is greater variation across direct developers in metamorphic re-

557 patterning than is modeled here. In some cases, the sequence of developmental events

558 is shortened because the formation of larval structures is lost from ontogeny. In other

559 cases, most or all events of embryogenesis and metamorphosis are retained but occur

560 inside the egg (which allows for the possibility of re-evolution of metamorphosis; Alberch

561 1989). We would predict more severe constraints in these latter lineages. Although we

562 treated both scenarios as a single category for simplicity, these two types of direct de-

563 velopment may be different in their effects on genome size evolution and warrant more

564 detailed study. 
566 Genome size evolution in paedomorphs.-Paedomorphic salamanders retain a larval

567 body form throughout life and show a deterministic trend towards genome expansion.

568 This trend is consistent with TE accumulation proceeding to higher overall levels, un-

569 checked by any constraints imposed by metamorphic repatterning. However, we do not

570 suggest that genome size is free from all constraints. The impacts of decreased sur-

571 face-area-to-volume ratio that accompany increased cell size likely impose an upper

572 limit on cell function that salamanders may well have reached (Chan and Marshall

573 2010); their cells are among the largest found in animals (Horner and Macgregor 1983).

574 In addition, the duration of embryogenesis may well have an upper bound that con-

575 strains genome expansion at the extremely high end. In the past, huge cells have been

576 proposed as adaptive because they coincide, at broad taxonomic levels, with low meta-

577 bolic rates; salamanders and lungfishes have the lowest metabolic rates and the largest

578 genomes/cells within vertebrates. This correlation led to the proposal that selection

579 shaped an adaptive "frugal metabolic strategy" in these taxa (Szarski 1983; Olmo, et al.

580 1989). More recent analyses of the relationship between genome/cell size and meta-

581 bolic rate, however, have failed to find a clear relationship (Licht and Lowcock 1991;

582 Uyeda, et al. 2017; Gardner, et al. 2020). Thus, empirical evidence that huge genomes

583 are a product of directional selection is currently lacking. Our results are more con-

584 sistent with the relaxation of a constraint against genome expansion because of the ex-

585 tremely weak deterministic pull strength and strong stochastic noise parameters.

587 Model complexity to capture the evolutionary process. - While the best model includes a 588 deterministic pull parameter, its magnitude is miniscule. Yet our model selection results 
demonstrate that models with deterministic pull provide a huge improvement over any purely stochastic model (Table 2). One of the challenges of an OU model with a weak

591 deterministic component is that the model parameters will be poorly defined (Cressler,

592 et al. 2015). Intuitively, if the pull parameter $(\alpha)$ is important but close to zero, the equi-

593 libria $(\theta i)$ can take on a wide range of values in combination with a range of values for

594 the stochastic parameter $\sigma$ and explain the phenotypic distribution equally as well. The 595 difference between a purely stochastic (BM) model and one that has any degree of de596 terministic pull is that the variance of a BM model will grow unbounded over time (as

597 variance of a trait undergoing a Brownian motion process is proportional to time),

598 whereas the variance in a model with deterministic pull will not (variance $\sim \sigma^{2} / 2 \alpha$ )

599 (Hansen and Martins 1996; Butler and King 2004). Thus, while the phenotype may ex-

600 plore a wide range of values in an OU model with very small $\alpha$, it will remain bounded.

601 For paedomorphs, a weak deterministic pull allows the mean to wander, while a far-

602 away equilibrium value captures a deterministic trend toward increase. In OU models,

603 increasing deterministic pull strength influences the approach to the equilibrium, but

604 also will tend to dampen stochastic effects (apart from the influence of $\sigma$ ), so it is possi-

605 ble to have both weak deterministic pull and substantial noise in the stochastic process.

606 This analysis demonstrates that deterministic pull can exert an important evolutionary

607 influence, even if the magnitude of alpha is weak.

608 But how complex a model is necessary? One might suppose that stronger deter-

609 ministic pull on one portion of the tree would support a rate shift in $\alpha$. However, all multi-

610 ple $\alpha$ models performed poorly. This is consistent with extensive simulation results 
611 showing that, among the three basic parameters of the OU model, $\alpha$ is most poorly de-

612 fined (Boettiger, et al. 2012; Ho and Ané 2013; Cressler, et al. 2015). Thus, even if a

613 rate shift in $\alpha$ existed, there is probably little power to detect it. In this study, we find that

614 with multiple free parameters, we can readily capture shifts in the evolutionary process

615 with variable $\theta$ and perhaps $\sigma$ over the tree, with stronger deterministic trends accom-

616 plished by moving $\theta$ to more extreme values. A shift in $\alpha$ is superlative, as changes in $\alpha$

617 and $\theta$ are not independently identifiable. Beyond cases with weak deterministic pull, dif-

618 ficulty in identifiability of $\alpha$ may be a general problem for all comparative studies, as we

619 do not know of a case as of yet where a multiple $\alpha$ model was superior.

620 What this analysis clearly illustrates is the importance of including relevant bio-

621 logically-informed hypotheses without over-parameterizing the evolutionary model. Iso-

622 lating the evolutionary "signal" from the "noise" in this dataset was most strongly aided

623 by including relevant hypotheses for variation in constraint, particularly by specifying

624 shifts in metamorphosis and non-feeding metamorphosis along the evolutionary history

625 of salamanders. When looking at adding parameters to the evolutionary model, the ef-

626 fects were variable with strongly positive $(\alpha)$, neutral (multiple $\sigma$ ), or strongly detri-

627 mental (multiple $\alpha$ ) effects on explanatory power. We note that the harm from overpa-

628 rameterizing is not only significantly poorer model scores, but at least in this dataset, the

629 order of the hypotheses changed. It is possible that these significantly worse-fit models

630 leak variation from some of the regime categories to the extraneous parameters. It is

631 critical for model testing, therefore, to include a test of all of the meaningful biological 
632 hypotheses with a range of parameterizations including models with fewer parameters.

634 Exploring evolutionary constraints with the comparative method.-Constraint has long

635 been argued as a necessary component of the evolutionary toolbox, as workers realized

636 that selection alone is insufficient to explain macroevolutionary shifts in phenotype

637 (Alberch 1980; Gould 1980; Cheverud 1984). The concept of constraint is paradoxically

638 both simple: "evolutionary constraints are restrictions or limitations on the course or out-

639 come of evolution" and wide-reaching, including genetic, selective, developmental, and

640 functional constraints (Arnold 1992). Many fields have attempted to quantify constraints

641 at various levels of biological organization using the notion of limitation. Genetic con-

642 straints have been identified as limitations on genetic variation (Kirkpatrick and Lofsvold

643 1992), constrained responses to selection (Cheverud 1984), or multivariate correlations

644 that are antagonistic to the direction of selection (Etterson and Shaw 2001). All of these

645 mechanisms allow the phenotype to change in ways that are not directly adaptive. De-

646 velopmental canalization, decisions in the developmental program which narrow the

647 phenotypic possibilities in some traits later in ontogeny, illustrate the potentially creative

648 force of constraint when coupled with modularity by opening opportunities for the larger

649 jumps in phenotype that become possible by evolutionary changes in modules (Wagner

650 1988; Wagner and Altenberg 1996; Jones, et al. 2018). Here, bounds to evolution

651 clearly extend the range of possible phenotypic evolution. In terms of broader compara-

652 tive study, modularity in multivariate shape evolution has been studied via patterns of

653 multivariate covariation, especially for skeletal features (Parsons, et al. 2012; Jones, et

654 al. 2018). However, progress in applying concepts of constraint on single characters 
655 has been hampered by a lack of precision in the concept of constraint and how it could 656 be detected.

Adaptation is typically envisioned as an evolutionary process whereby pheno-

658 types evolve in response to the strong pull toward an optimum, which tends to concen-

659 trate phenotypes about one or more optima after sufficient time has proceeded. On the

660 other hand, an evolutionary process bounded by constraint would be dominated by sto-

661 chastic evolution for much of the range of a phenotype, appearing as random evolution

662 with a substantially larger — but bounded — variance. Only when the phenotype

663 crosses a threshold would selection come into play, and this may occur due to corre-

664 lated selection on another linked trait. Thus, the evolutionary "driving force" can simply

665 be stochastic change with limits imposed by constraints. Our results support this theo-

666 retical model as an explanation for genome size evolution in salamanders. More gener-

667 ally, our results show that OU models, which incorporate both the change in the mean

668 as well as variance of the phenotype, can be used to distinguish between trait evolution

669 explained by a strong pull toward an optimum versus weak selection with bounded vari-

670 ance. Phylogenetic comparative methods have by and large focused on explaining

671 shifts in mean phenotype, and have thus lent themselves well to studying adaptation,

672 convergent evolution, and parallelism. By diving deeper into the exploration of stochas-

673 tic models with opportunities for varying selection and noise, we can make great pro-

674 gress in understanding the action of constraint in shaping evolution.

676 Salamanders as a model for linking organismal traits to genome biology.-Much re-

677 mains to be learned about how selection on organismal traits translates into changes in 
678 genome size, and this represents a gap in our knowledge of genome biology and evolu-

679 tion that studies of salamanders can help to fill. For example, our results suggest that

680 salamanders that undergo non-feeding metamorphosis can be leveraged as a model

681 system to distinguish between possible mechanisms of genome reduction. Variation in

682 genome size is introduced into a population by TE insertions and deletions. Selection

683 could, in principle, sort among these genome size variants, even as the fitness conse-

684 quences of individual TE loci are typically miniscule and effectively neutral (Lynch 2007;

685 Arkhipova 2018). In addition, selection could sort among differences in TE control ma-

686 chinery including the pathways that underlie TE silencing and deletion, which could yield

687 variants with greater differences in TE composition and fitness (Mueller 2015; Parhad

688 and Theurkauf 2019; Parhad, et al. 2020). Comparing TE control machinery in non-

689 feeding metamorphosers versus other life history regimes (e.g. paedomorphs) could

690 identify the mechanisms underlying genome reduction.

691

692 Conclusion. - Overall, our study shows that the evolution of a life history that includes

693 non-feeding metamorphosis exerts a unique influence on the evolution of genome size,

694 imposing the most severe constraints of any salamander life history strategy. This result

695 suggests that selection to shorten the duration of metamorphosis because of energetic

696 vulnerability has indirectly selected for faster development, constraining cells and ge-

697 nomes to smaller sizes. We show that genome size evolution is shaped by strong sto-

698 chastic forces that are not widely variable across the salamander clade, but that deter-

699 ministic forces — which vary enormously across life histories — have also played an im- 
700 portant role. We broaden the application of stochastic models of trait evolution to in-

701 clude constraint, and we highlight the challenges inherent in connecting model parame-

702 ters and parameter values to complex biological phenomena.

703

704

705

706

Literature Cited

707

Alberch P. 1989. Development and the evolution of amphibian metamorphosis. In:

708 Splechtna/Hilgers, editor. Trends in Vertebrate Morphology. Stuttgart: Gustav Fischer

709 Verlag. p. 163-173.

Alberch P. 1980. Ontogenesis and morphological diversification. Am Zool 20:653-667.

711 AmphibiaWeb: Information on amphibian biology and conservation [Internet]. 2021.

712 Berkeley, California. Available from http://amphibiaweb.org/.

713 Arkhipova IR. 2018. Neutral theory, transposable elements, and eukaryotic genome

714 evolution. Mol Biol Evol 35:1332-1337.

715 Arnold SJ. 1992. Constraints on phenotypic evolution. Am Nat 140:S85-S107.

716 Arnold SJ, Wassersug RJ. 1978. Differential predation on metamorphic anurans by

717 garter snakes (Thamnophis): Social behavior as a possible defense. Ecology 59:1014-

7181022.

719 Beachy CK, Ryan TJ, Bonett RM. 2017. How metamorphosis is different in

720 plethodontids: Larval life history perspectives on life-cycle evolution. Herpetologica

$721 \quad 73: 252-258$. 
722 Beaulieu JM, Jhwueng D-C, Boettiger C, O’Meara BC. 2012. Modeling stabilizing

723 selection: Expanding the Ornstein-Uhlenbeck model of adaptive evolution. Evolution

724 66:2369-2383.

725 Boettiger C, Coop G, Ralph P. 2012. Is your phylogeny informative? Measuring the 726 power of comparative methods. Evolution 66:2240-2251.

727 Bonett RM. 2016. An integrative endocrine model for the evolution of developmental

728 timing and life history of plethodontids and other salamanders. Copeia 104:209-221.

729 Bonett RM, Hess AJ, Ledbetter NM. 2020. Facultative transitions have trouble

730 committing, but stable life cycles predict salamander genome size evolution. Evol Biol

$731 \quad 47: 111-122$.

732 Bonett RM, Mueller RL, Wake DB. 2005. Why should reaquisition of larval stages by

733 Desmognathine salamanders surprise us? Herpetol Rev 36:112.

734 Bonett RM, Steffen MA, Robison GA. 2014. Heterochrony repolarized: a phylogenetic 735 analysis of developmental timing in plethodontid salamanders. Evo Devo 5:27.

736 Butler Marguerite A, King Aaron A. 2004. Phylogenetic comparative analysis: A

737 modeling approach for adaptive evolution. Am Nat 164:683-695.

738 Carta A, Bedini G, Peruzzi L. 2020. A deep dive into the ancestral chromosome number

739 and genome size of flowering plants. New Phytol 228:1097-1106.

740 Chan Y-HM, Marshall WF. 2010. Scaling properties of cell and organelle size.

741 Organogenesis 6:88-96. 
742 Cheverud JM. 1984. Quantitative genetics and developmental constraints on evolution

743 by selection. J Theor Biol 110:155-171.

744 Cheverud JM, Dow MM, Leutenegger W. 1985. The quantitative assessment of

745 phylogenetic constraints in comparative analyses: Sexual dimorphism in body weight

746 among primates. Evolution 39:1335-1351.

747 Chippindale PT, Bonett RM, Baldwin AS, Wiens JJ. 2004. Phylogenetic evidence for a 748 major reversal of life-history evolution in plethodontid salamanders. Evolution 58:28097492822.

750 Cooper N, Thomas GH, Venditti C, Meade A, Freckleton RP. 2016. A cautionary note 751 on the use of Ornstein Uhlenbeck models in macroevolutionary studies. Biol J Linn Soc 752 118:64-77.

753 Cressler CE, Butler MA, King AA. 2015. Detecting adaptive evolution in phylogenetic 754 comparative analysis using the Ornstein-Uhlenbeck model. Syst Biol 64:953-968.

755 Deban SM, Marks SB. 2002. Metamorphosis and evolution of feeding behaviour in 756 salamanders of the family Plethodontidae. Zool J Linn Soc 134:375-400.

757 Decena-Segarra LP, Bizjak-Mali L, Kladnik A, Sessions SK, Rovito SM. 2020.

758 Miniaturization, genome size, and biological size in a diverse clade of salamanders. Am 759 Nat 196:634-648.

760 Downie JR, Bryce R, Smith J. 2004. Metamorphic duration: an under-studied variable in 761 frog life histories. Biol J Linn Soc 83:261-272.

762 Edgar RC. 2004. MUSCLE: multiple sequence alignment with high accuracy and high 763 throughput. Nucleic Acids Res 32:1792-1797. 
764 Elliott TA, Gregory TR. 2015. What's in a genome? The C-value enigma and the

765 evolution of eukaryotic genome content. Philos Trans R Soc B: Biol Sci 370:20140331.

766 Etterson JR, Shaw RG. 2001. Constraint to adaptive evolution in response to global 767 warming. Science 294:151.

768 Felsenstein J. 1985. Phylogenies and the comparative method. Am Nat 125:1-15.

769 Frahry MB, Sun C, Chong R, Mueller RL. 2015. Low levels of LTR retrotransposon

770 deletion by ectopic recombination in the gigantic genomes of salamanders. J Mol Evol

$77180: 120-129$.

772 Gardner JD, Laurin M, Organ CL. 2020. The relationship between genome size and

773 metabolic rate in extant vertebrates. Philos Trans R Soc B 375:20190146.

774 Gould SJ. 1980. Is a new and general theory of evolution emerging? Paleobiology

775 6:119-130.

776 Gould SJ. 1977. Ontogeny and Phylogeny. Cambridge, MA: Harvard University Press.

777 Grafen A, Hamilton WD. 1989. The phylogenetic regression. Philosophical Transactions

778 of the Royal Society of London. B, Biological Sciences 326:119-157.

779 Gregory TR. 2002a. A bird's eye view of the c-value enigma: genome size, cell size, and 780 metabolic rate in the class Aves. Evolution 56:121-130.

781 Gregory TR. 2001. Coincidence, coevolution, or causation? DNA content, cell size, and 782 the C-value enigma. Biol Rev Camb Philos Soc 76:65-101.

783 Gregory TR. 2005. The Evolution of the Genome. San Diego, CA: Academic Press. 
784 Gregory TR. 2002b. Genome size and developmental complexity. Genetica 115:131785146.

786 Gregory, T. R. Animal Genome Size Database (http://www.genomesize.com) [Internet]. 7872021.

788 Hansen TF. 1997. Stabilizing selection and the comparative analysis of adaptation.

789 Evolution 51:1341-1351.

790 Hansen TF, Martins EP. 1996. Translating between microevolutionary process and

791 macroevolutionary patterns: The correlation structure of interspecific data. Evolution $792 \quad 50: 1404-1417$.

793 Hessen DO, Daufresne M, Leinaas HP. 2013. Temperature-size relations from the 794 cellular-genomic perspective. Biol Rev 88:476-489.

795 Ho LST, Ané C. 2013. Asymptotic theory with hierarchical autocorrelation: Ornstein796 Uhlenbeck tree models. Ann Statist 41:957-981.

797 Ho LST, Ané C. 2014. Intrinsic inference difficulties for trait evolution with Ornstein798 Uhlenbeck models. Methods Ecol Evol 5:1133-1146.

799 Horner HA, Macgregor HC. 1983. C value and cell volume: their significance in the 800 evolution and development of amphibians. J Cell Sci 63:135.

801 Huey RB, Bennett AF. 1987. Phylogenetic studies of coadaptation: preferred

802 temperatures versus optimal performance temperatures of lizards. Evolution 41:10988031115. 
804 Jeffery NW, Yampolsky L, Gregory TR. 2016. Nuclear DNA content correlates with

805 depth, body size, and diversification rate in amphipod crustaceans from ancient Lake

806 Baikal, Russia. Genome 60:303-309.

807 Jockusch EL. 1997. An evolutionary correlate of genome size change in plethodontid

808 salamanders. Proc Royal Soc B: Biol Sci 264:597.

809 Jones KE, Benitez L, Angielczyk KD, Pierce SE. 2018. Adaptation and constraint in the

810 evolution of the mammalian backbone. BMC Evol Biol 18:172.

811 Keinath MC, Timoshevskiy VA, Timoshevskaya NY, Tsonis PA, Voss SR, Smith JJ.

812 2015. Initial characterization of the large genome of the salamander Ambystoma

813 mexicanum using shotgun and laser capture chromosome sequencing. Sci Rep

$814 \quad 5: 16413$.

815 Kerney RR, Blackburn DC, Müller H, Hanken J. 2012. Do larval traits re-evolve?

816 Evidence from the embryogenesis of a direct-developing salamander, Plethodon

817 cinereus. Evolution 66:252-262.

818 King Aaron A, Butler Marguerite A. 2009. OUCH: Ornstein-Uhlenbeck models for

819 phylogenetic comparative hypotheses (R package).

820 Kirkpatrick M, Lofsvold D. 1992. Measuring selection and constraint in the evolution of

821 growth. Evolution 46:954-971.

822 Landberg T, Azizi E. 2010. Ontogeny of escape swimming performance in the spotted

823 salamander. Funct Ecol 24:576-587.

824 Lande R. 1980. Genetic variation and phenotypic evolution during allopatric speciation.

825 Am Nat 116:463-479. 
826 Licht LE, Lowcock LA. 1991. Genome size and metabolic rate in salamanders. Comp

827 Biochem Physiol B: Comp Biochem 100:83-92.

828 Liedtke HC, Gower DJ, Wilkinson M, Gomez-Mestre I. 2018. Macroevolutionary shift in

829 the size of amphibian genomes and the role of life history and climate. Nat Ecol Evol

$830 \quad 2: 1792-1799$.

831 Lowe WH, Swartz LK, Addis BR, Likens GE. 2019. Hydrologic variability contributes to

832 reduced survival through metamorphosis in a stream salamander. Proc Natl Acad Sci

833 USA 116:19563.

834 Lynch M. 2007. The Origins of Genome Architecture. Sunderland, MA: Sinauer

835 Associates, Inc.

836 Maddison WP. 1991. Squared-change parsimony reconstructions of ancestral states for

837 continuous-valued characters on a phylogenetic tree. Syst Biol 40:304-314.

838 Madison-Villar MJ, Sun C, Lau N, Settles M, Mueller RL. 2016. Small RNAs from a big

839 genome: the piRNA pathway and transposable elements in the salamander species

840 Desmognathus fuscus. J Mol Evol 83:126-136.

841 Michael TP. 2014. Plant genome size variation: bloating and purging DNA. Brief Funct

842 Genom 13:308-317.

843 Mohlhenrich E, Mueller RL. 2016. Genetic drift, mutational hazard, and the evolution of 844 genomic gigantism in salamanders. Evolution 70:2865-2878.

845 Moran NA. 1994. Adaptation and constraint in the complex life cycles of animals. Ann 846 Rev Ecol Syst 25:573-600. 
847 Mueller RL. 2015. Genome biology and the evolution of cell size diversity. In: Heald R,

848 Hariharan I, Wake DB, editors. Size Control in Biology, from Organelles to Organisms:

849 Cold Spring Harbor Press.

850 Mueller RL, Gregory TR, Gregory SM, Hsieh A, Boore JL. 2008. Genome size, cell size, 851 and the evolution of enucleated erythrocytes in attenuate salamanders. Zoology

$852 \quad 111: 218-230$.

853 Mueller RL, Macey JR, Jaekel M, Wake DB, Boore JL. 2004. Morphological homoplasy, 854 life history evolution, and historical biogeography of plethodontid salamanders inferred 855 from complete mitochondrial genomes. Proc Natl Acad Sci USA 101:13820-13825.

856 Norman MF. 1985. A practical method for staging metamorphosis in the tiger 857 salamander Ambystoma tigrinum. Anatom Rec 211:102-109.

858 Nowoshilow S, Schloissnig S, Fei J-F, Dahl A, Pang AWC, Pippel M, Winkler S, Hastie 859 AR, Young G, Roscito JG, et al. 2018. The axolotl genome and the evolution of key 860 tissue formation regulators. Nature 554:50-55.

861 O'Meara BC, Ané C, Sanderson MJ, Wainwright PC. 2006. Testing for different rates of 862 continuous trait evolution using likelihood. Evolution 60:922-933.

863 Olmo E, Capriglione T, Odierna G. 1989. Genome size evolution in vertebrates: Trends 864 and constraints. Comp Biochem Phys B: Comp Biochem 92:447-453.

865 Orlofske SA, Hopkins WA. 2009. Energetics of metamorphic climax in the pickerel frog 866 (Lithobates palustris). Comp Biochem Phys A: Molec Integ Phys 154:191-196. 
867 Pandit MK, White SM, Pocock MJO. 2014. The contrasting effects of genome size, 868 chromosome number and ploidy level on plant invasiveness: a global analysis. New 869 Phytol 203:697-703.

870 Parhad SS, Theurkauf WE. 2019. Rapid evolution and conserved function of the piRNA 871 pathway. Roy Soc Open Biol 9:180181.

872 Parhad SS, Yu T, Zhang G, Rice NP, Weng Z, Theurkauf WE. 2020. Adaptive evolution 873 targets a piRNA precursor transcription network. Cell Rep 30:2672-2685.e2675.

874 Parsons KJ, Márquez E, Albertson RC. 2012. Constraint and opportunity: The genetic 875 basis and evolution of modularity in the cichlid mandible. Am Nat 179:64-78.

876 Pasquesi GIM, Adams RH, Card DC, Schield DR, Corbin AB, Perry BW, Reyes-Velasco

877 J, Ruggiero RP, Vandewege MW, Shortt JA, et al. 2018. Squamate reptiles challenge

878 paradigms of genomic repeat element evolution set by birds and mammals. Nat

879 Commun 9:2774.

880 Posada D, Crandall KA. 1998. MODELTEST: testing the model of DNA substitution.

881 Bioinformatics 14:817-818.

882 Pyron AR, Wiens JJ. 2011. A large-scale phylogeny of Amphibia including over 2800 883 species, and a revised classification of extant frogs, salamanders, and caecilians. Mol 884 Phylogenet Evol 61:543-583.

885 R Core Team. 2020. R: A language and environment for statistical computing. Vienna, 886 Austria: R Foundation for Statistical Computing.

887 Roddy AB, Théroux-Rancourt G, Abbo T, Benedetti JW, Brodersen CR, Castro M, 888 Castro S, Gilbride AB, Jensen B, Jiang G-F, et al. 2019. The scaling of genome size 
889 and cell size limits maximum rates of photosynthesis with implications for ecological

890 strategies. Int J Plant Sci 181:75-87.

891 Rose CS. 2014. The importance of cartilage to amphibian development and evolution.

892 Int J Dev Biol 58:917-927.

893 Rose CS. 1995a. Skeletal morphogenesis in the urodele skull: II. Effect of

894 developmental stage in thyroid hormone-induced remodeling. J Morph 223:149-166.

895 Rose CS. 1995b. Skeletal morphogenesis in the urodele skull: Ill. Effect of hormone 896 dosage in th-induced remodeling. J Morph 223:243-261.

897 Rose CS. 1995c. Skeletal morphogenesis in the urodele skull:I. Postembryonic

898 development in the hemidactyliini (amphibia: Plethodontidae). J Morph 223:125-148.

899 Sanchez E, Küpfer E, Goedbloed DJ, Nolte AW, Lüddecke T, Schulz S, Vences M,

900 Steinfartz S. 2018. Morphological and transcriptomic analyses reveal three discrete

901 primary stages of postembryonic development in the common fire salamander,

902 Salamandra salamandra. J Exp Zool B: 330:96-108.

903 Sanderson MJ. 2003. r8s: inferring absolute rates of molecular evolution and

904 divergence times in the absence of a molecular clock. Bioinformatics 19:301-302.

905 Schluter D, Price T, Mooers AØ, Ludwig D. 1997. Likelihood of ancestor states in 906 adaptive radiation. Evolution 51:1699-1711.

907 Sessions SK. 2008. Evolutionary cytogenetics in salamanders. Chromosome Res 908 16:183-201. 
909 Shao F, Han M, Peng Z. 2019. Evolution and diversity of transposable elements in fish

910 genomes. Sci Rep 9:15399.

911 Simpson GG. 1953. The Major Features of Evolution. New York: Columbia University 912 Press.

913 Smith JDL, Bickham JW, Gregory TR. 2013. Patterns of genome size diversity in bats

914 (order Chiroptera). Genome 56:457-472.

915 Sotero-Caio CG, Platt RN, II, Suh A, Ray DA. 2017. Evolution and diversity of

916 transposable elements in vertebrate genomes. Genome Biol Evol 9:161-177.

917 Stamatakis A. 2006. RAxML-VI-HPC: maximum likelihood-based phylogenetic analyses

918 with thousands of taxa and mixed models. Bioinformatics 22:2688-2690.

919 Sun C, Arriaza JRL, Mueller RL. 2012. Slow DNA loss in the gigantic genomes of

920 salamanders. Genome Biol Evol 4:1340-1348.

921 Sun C, Mueller RL. 2014. Hellbender genome sequences shed light on genome

922 expansion at the base of crown salamanders. Genome Biol Evol 6:1818-1829.

923 Sun C, Shepard DB, Chong RA, Arriaza JL, Hall K, Castoe TA, Feschotte C, Pollock

924 DD, Mueller RL. 2012. LTR retrotransposons contribute to genomic gigantism in

925 plethodontid salamanders. Genome Biol Evol 4:168-183.

926 Szarski H. 1983. Cell size and the concept of wasteful and frugal evolutionary

927 strategies. J Theor Biol 105:201-209.

928 Uyeda JC, Pennell MW, Miller ET, Maia R, McClain CR. 2017. The evolution of

929 energetic scaling across the vertebrate tree of life. Am Nat 190:185-199. 
930 Vieites DR, Rom·n SN, Wake MH, Wake DB. 2011. A multigenic perspective on

931 phylogenetic relationships in the largest family of salamanders, the Plethodontidae. Mol

932 Phylogenet Evol 59:623-635.

933 Vinogradov AE. 2004. Genome size and extinction risk in vertebrates. Proc Roy Soc B 934 271:1701-1705.

935 Vladimirova IG, Kleimenov SY, Alekseeva TA. 2012. Dynamics of body mass and

936 oxygen consumption in the ontogeny of the Spanish ribbed newt (Pleurodeles waltl): 2.

937 Larval stage. Biol Bull 39:10-14.

938 Vu GTH, Cao HX, Reiss B, Schubert I. 2017. Deletion-bias in DNA double-strand break 939 repair differentially contributes to plant genome shrinkage. New Phytol 214:1712-1721.

940 Wagner GP. 1988. The significance of developmental constraints for phenotypic

941 evolution by natural selection. In: de Jong G, editor. Population Genetics and Evolution.

942 Berlin, Heidelberg: Springer.

943 Wagner GP, Altenberg L. 1996. Perspective: Complex adaptations and the evolution of 944 evolvability. Evolution 50:967-976.

945 Wake D, Hanken J. 2004. Direct development in the lungless salamanders: what are 946 the consequences for developmental biology, evolution and phylogenesis? Int J Dev 947 Biol 40:859-869.

948 Wake DB, Marks SB. 1993. Development and evolution of plethodontid salamanders: a 949 review of prior studies and a prospectus for future research. Herpetologica 49:194-203.

950 Waltari E, Edwards SV. 2002. Evolutionary dynamics of intron size, genome size, and 951 physiological correlates in archosaurs. Am Nat 160:539-552. 
952 Wassersug RJ, Sperry DG. 1977. The relationships of locomotion to differential

953 predation on Pseudacris triseriata (anura: Hylidae). Ecology 58:830-839.

954 Wiens JJ, Bonett RM, Chippindale PT. 2005. Ontogeny discombobulates phylogeny:

955 paedomorphosis and higher-level salamander relationships. Syst Biol 54:91-110.

956 Wright ML, Richardson SE, Bigos JM. 2011. The fat body of bullfrog (Lithobates

957 catesbeianus) tadpoles during metamorphosis: Changes in mass, histology, and

958 melatonin content and effect of food deprivation. Comp Biochem Phys A 160:498-503.

959 Zheng Y, Peng R, Kuro-o M, Zeng X. 2011. Exploring patterns and extent of bias in

960 estimating divergence time from mitochondrial DNA sequence data in a particular

961 lineage: a case study of salamanders (Order Caudata). Mol Biol Evol 28:2521-2535. 